\title{
Growth Phase of Magnetospheric Substorms
}

\author{
Robert L. McPherron \\ Institute of Geophysics and Planetary Physics \\ University of California, Los Angeles 90024
}

The term 'magnetospheric substorm' has been used recently to describe the collection of phenomena that occur throughout the magnetosphere at times of auroral and magnetic substorm activity [e.g., Brice, 1967; McPherron et al., 1967; Coroniti et al., 1968]. This concept is an extension of the ideas of Akasofu and co-workers, who through extensive surface observations of auroral and magnetic disturbances developed models of the auroral substorm [Akasofu, 1964] and the polar magnetic substorm [Akasofu et al., 1965; Akasofu et al., 1966a; Akasofu and Meng, 1969]. In these phenomenological models the authors were able to show that various features of the aurora and magnetic disturbance could be organized if geomagnetic latitude, local time, and time from the onset of the event were considered.

Previous models of substorms have consisted of two main phases, an explosive expansion phase and a more gradual recovery phase. The interval between isolated substorms was called the 'quiet phase' [Akasofu, 1964]. Evidence presented in this paper suggests that polar magnetic substorms have a significant growth phase prior to the expansion phase. Previous reports in the literature reveal that many magnetospheric phenomena occur at the same time as the growth phase. It thus seems appropriate to expand the concept of a magnetospheric substorm to include such a growth phase.

\section{Experimental Results}

Characteristic features of polar magnetic substorms. The model of a polar magnetic substorm provides a particularly convenient means of analyzing magnetograms to determine the universal time at which a substorm has occurred. Magnetogram signatures of a substorm used in this analysis are described below.

Copyright @ 1970 by the American Geophysical Union.
According to Akasofu et al. [1966a], the substorm has two characteristic phases, an expansion phase and a recovery phase. For magnetic observatories near midnight a northward expansion of auraral and magnetic disturbance causes an obvious change in sign of the vertical component as the disturbance passes overhead [Akasofu et al., 1966b]. Similarly, a station several hours before midnight experiences a very sudden decrease in the horizontal component as the westward surge passes overhead [Akasofu et al., 1966a]. Stations still earlier, near dusk in a region normally dominated by positive disturbances in the horizontal component, experience a negative indentation [Meng and Akasofu, 1967] as the surge carries the region of magnetic disturbance westward.

The end of the expansion occurs approximately $30 \mathrm{~min}$ after its beginning. The characteristic features in magnetograms at this time are extremes in the deviations of various components from their quiet values. This phase is followed by a recovery phase during which the 'auroral and magnetic disturbances decay.

\section{OBSERVATIONS}

Event of September 6, 1966. An example of a substorm with an obvious growth phase is shown in Figure 1. This figure contains tracings of the horizontal component at a number of auroral zone observatories. Vertical arrows at the left edge correspond to $500 \gamma$. The quiet levels indicated for each station by dashed lines are tracings of the magnetic variations recorded on the quiet day, August 28, 1966. Magnetic midnight at each station is indicated by the letter $\mathrm{M}$ below the corresponding trace.

The substorm growth phase began about 0100 UT on September 6, after about 6 hours with no substorm activity. It is not possible to determine this start time exactly because the growth phase is initially characterized by small 


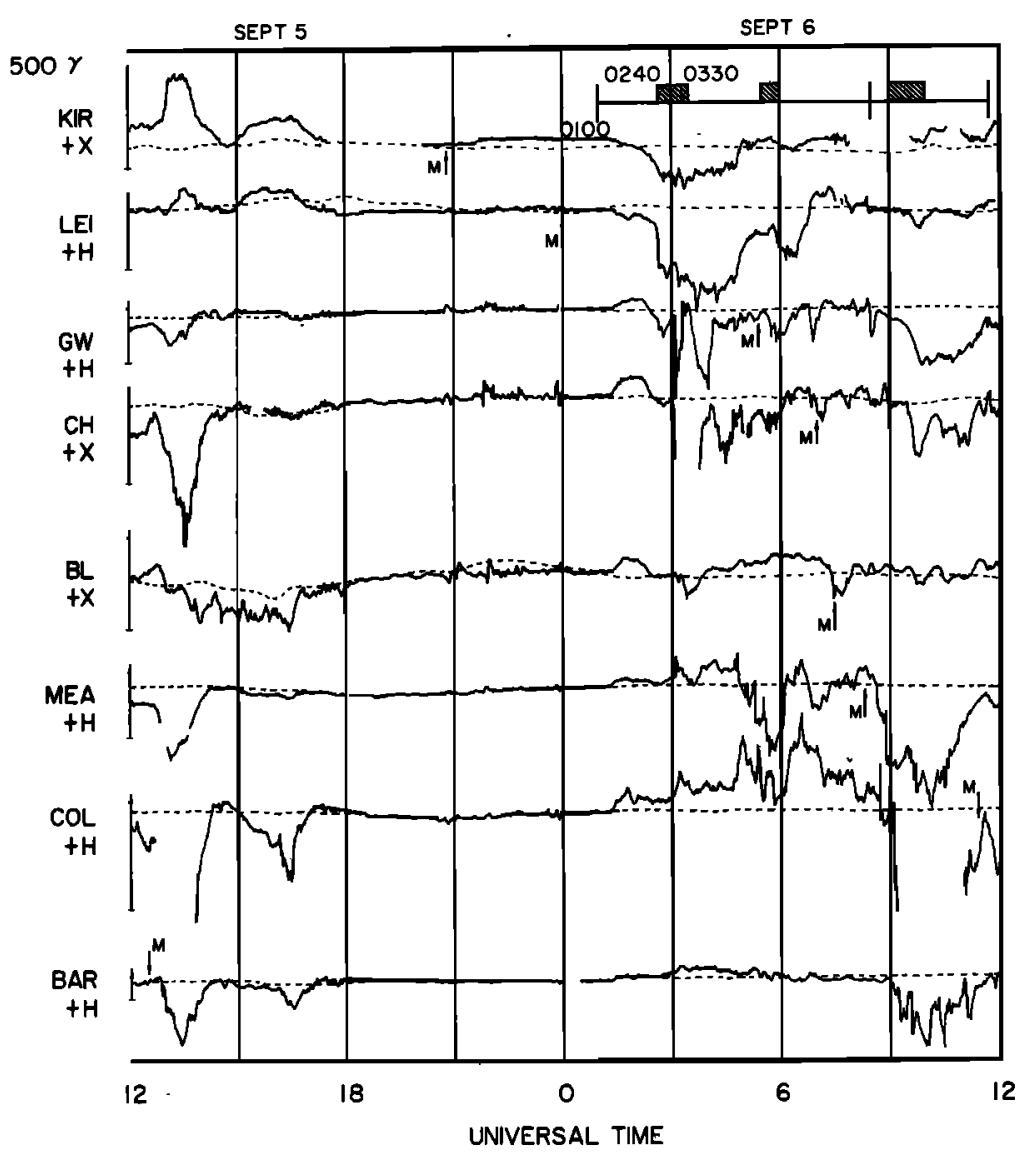

Fig. 1. Horizontal component of magnetic field variation at a number of auroral zone stations during a polar magnetic substorm at 0240 UT, September 1966.

deviations from the quiet trace. Consequently, the exact start time depends on the choice of a quiet day and the definition of a significant deviation. However, shortly after 0100 , deviations larger than any in the preceding 6 hours occurred at all stations.

The sense of the deviation of the horizontal component during the growth phase is the same in various local time sectors as in later stages of the substorm. For example, in Figure 1 Kiruna (KIR) and Leirvogur (LEI) (top of figure) were located in the morning sector (note arrow indicating magnetic midnight). Both of these stations recorded a negative deviation. In contrast, College (COL) and Barrow (BAR) (bottom of figure) were in the afternoon and positive deviations were recorded. The remaining stations, Great Whale (GW), Fort Churchill (CH), Baker Lake (BL), and Meanook (MEA), were in the evening sector. The initial deviation at each of these stations was also positive.

The end of the growth phase of this substorm occurred between 0240 and 0300 UT depending on the location of the station. A sharp drop in the $H$ component at Leirvogur suggests that the expansion phase of a substorm began about 0240 UT. The westward surge carried the effects of this expansion toward dusk, and they appear at Great Whale and Fort Churchill about 0300 UT.

On a basis of this example, we conclude that following intervals of magnetic calm the horizontal component of the magnetic field experiences significant deviations prior to the start of the expansion phase of a magnetospheric substorm. Because the sign of the deviations in various local time sectors does not differ from that in later stages of the substorm, we also 


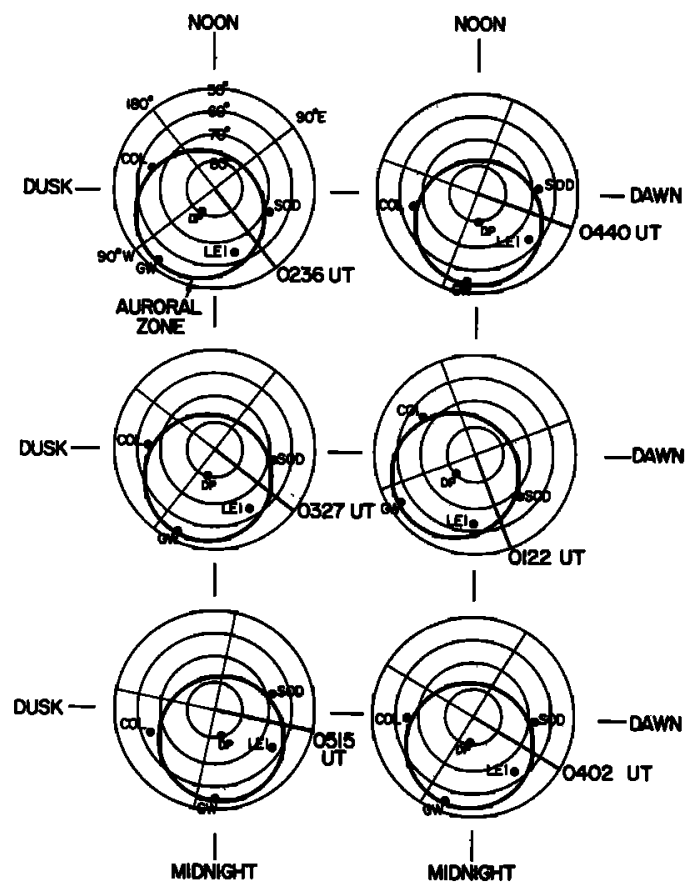

Fig. 2. Location of magnetic observatories in a north polar geographic projection at times corresponding to the start of the expansion phase of substorms shown in following figures. Note the universal time of expansion at the Greenwich meridian.

conclude that these deviations are likely to be caused by the same current systems. Consequently, it seems appropriate to name this initial part of a substorm the 'growth phase.'

Further examples of substorm growth phases. To check the conclusions discussed above, a number of additional events have been examined. Criteria for choosing these included the following to avoid confusion with effects of other substorms. The substorm should be an isolated event, or at the least be preceded by about 6 hours of magnetic calm. To assure a good distribution of auroral zone observatories, the substorm expansion should occur between 0100 and 0600 UT (local midnight in east-central Canada). Six events satisfying these criteria are discussed below.

The location of the four magnetic observatories used in the additional study are shown in north polar geographic projections in Figure 2. Noon is at the top and dawn at the right of each diagram. Individual diagrams illustrate the relative location of each station with respect to local midnight at the universal time of the start of each substorm expansion. Heavy lines indicate the auroral zone and the Greenwich meridian.

All three components of the magnetic field variation at each of the stations are shown in Figures 3, 4, and 5 for the six events. These figures were obtained by tracing optical enlargements of full-scale magnetograms to a scale of 1 inch per hour. All components are positive upward except College $D$. Base lines for studying substorm deviations were obtained by linear extrapolation of the trend in each component in the quiet hours preceding the substorm. These base lines are indicated by horizontal dashed lines.

The start times for each substorm were obtained in the following manner. A significant deviation of a component from its base line was defined as a variation greater than any in the quiet hours preceding the substorm. The substorm start time at each station was then defined as the time at which two or more components began significant deviations. Finally, the start time of the substorm was chosen as the time closest to the start time at all four stations. The resultant time is indicated on the figures by the vertical dashed line closest to the left edge of the figure.

The start time for each substorm expansion was obtained by a different procedure. The most distinct characteristic of a substorm expansion is the sudden sharp decrease in the horizontal component as the westward surge passes over a station just before local midnight. Thus, the start time of the expansion was defined as the time at which this decrease began for the station closest to midnight in the premidnight sector. These lines are also indicated by vertical dashed lines in the figures.

These definitions are subject to some error. For example, a more stringent definition of significant deviation would require choosing a later time as the start of a substorm. Further, if the closest pre-midnight station is some distance toward dusk, it is likely that the expansion began before the identified time. Both of these effects shorten the duration of the proposed growth phase. However, examination of the data presented below clearly shows that no 

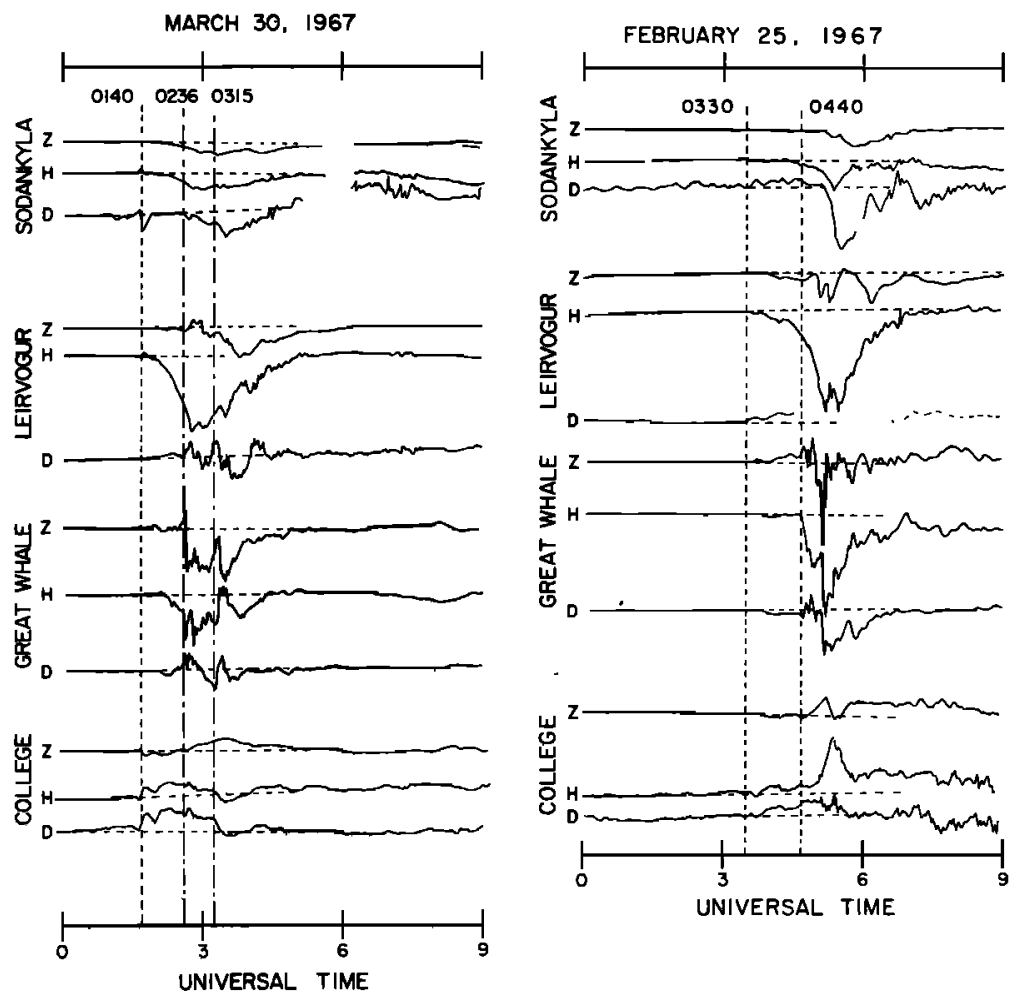

Fig. 3. All three components of substorm magnetic field variation at four observatories on February 25 and March 30, 1967. The leftmost vertical dashed line is the most probable time of the start of the substorm. The next vertical dashed line is the most probable time of the start of the substorm expansion.

reasonable definition of these two times can make them coincident. Consequently, we conclude that the duration of the growth phase may contain considerable error, but its existence is clearly shown.

A detailed examination of Figure 2 shows that in every event Sodankyla (SOD) is near dawn and College (COL) is near dusk. In all but the fourth event (0122 UT), Leirvogur (LEI) is in the predawn sector and Great Whale $(\mathrm{GW})$ is just before midnight.

Keep these facts in mind as we examine Figures 3, 4, and 5. Consider first the $H$ component at Sodankyla during the interval between the start of the substorm and the start of the expansion as defined above. In all six events the deviation is negative. Similarly, consideration of the College $H$ component reveals that in all six events the deviation is positive. These are exactly the sense of the more pronounced deviations that occur during the expansion and recovery phases of the substorm.

A similar study of the data for Leirvogur shows that in all events except perhaps event 5 (0515 UT of Figure 5) the deviation in $H$ is negative during this interval of time. Finally, we note that the $H$ component at Great Whale is generally positive first and then negative, as expected, for a station in the transition region between positive and negative bays.

The above observations support the conclusion stated at the end of the discussion of the September 6, 1966, substorm event, i.e., substorms have a significant growth phase. In the discussion presented below we show that a number of other magnetospheric phenomena also occur during this growth phase. Consequently, we conclude that these data simply indicate one of many manifestations of the growth phase of a magnetospheric substorm. 

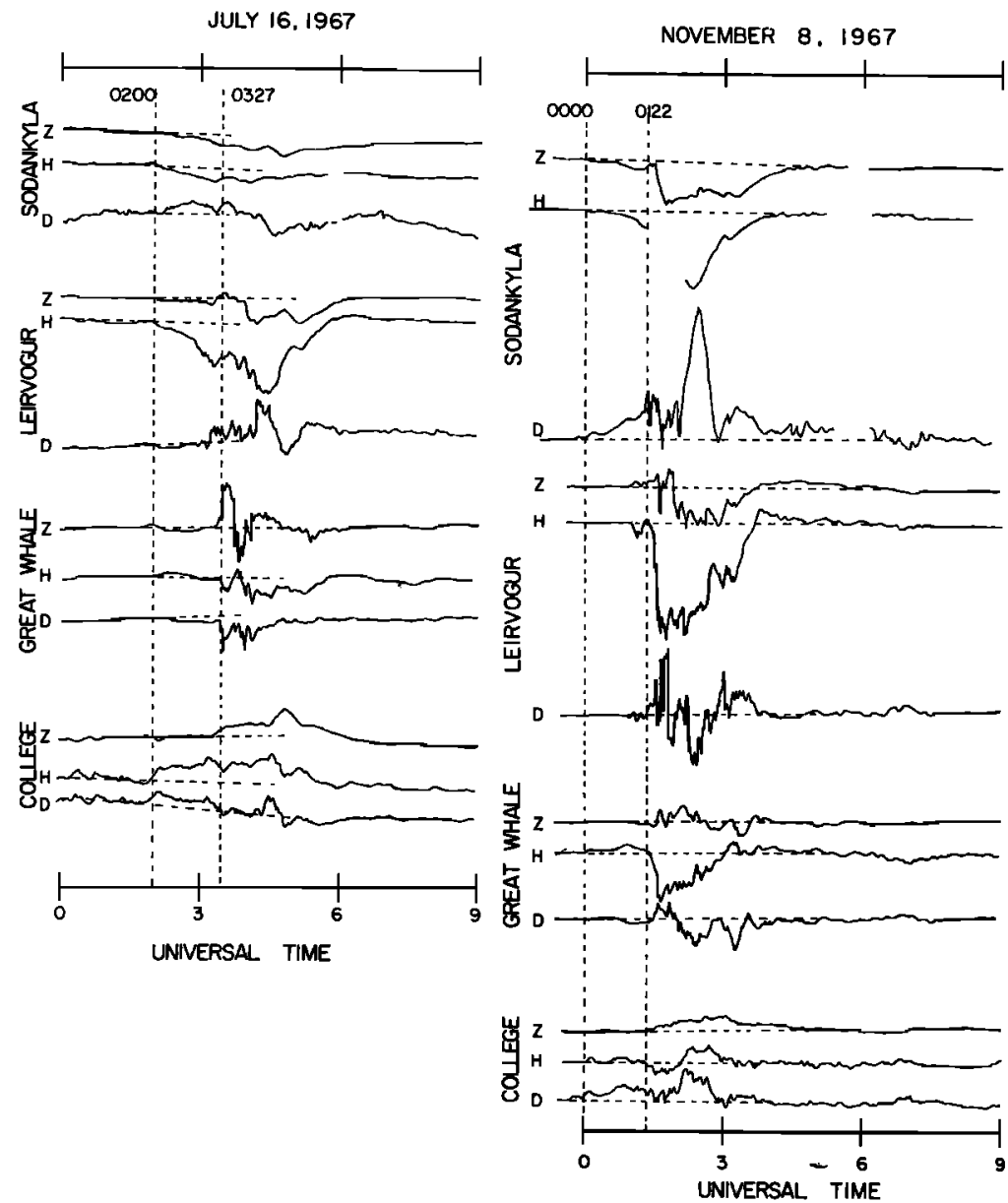

Fig. 4. Same as Figure 3 for July 16 and November 8, 1967.

\section{Discussion}

Magnetospheric phenomena associated with the growth phase. The data presented in the previous section establish the existence of the growth phase of a polar magnetic substorm. Because of the close association between the auroral ionosphere and the outer magnetosphere, it is probable that the growth phase should also be evident in magnetospheric phenomena. To establish this, it is important to review previous reports of magnetospheric phenomena associated with the substorm growth phase.

From ATS 1 magnetic field variations at 6.6 $R_{s}$ during substorms Cummings and Coleman [1968] and Cummings et al. [1968] have concluded that a partial ring current develops on the night side of the earth during substorms. They have reported that 1 to 4 hours before the onset of the expansion phase, the $H$ component at ATS begins a gradual decrease below quiet day values. A minimum is reached within an hour. The field may remain depressed for as long as 3 hours. At the time of the expansion phase as determined by ground magnetograms, the $H$ component at ATS 1 recovers abruptly all or a substantial fraction of the preceding decrease.

Vasyliunas [1968] has concluded from a study of low-energy electrons that inward convection of plasma is occurring during substorms. He interprets a steep temperature gradient of the low-energy electrons as the inner edge of the plasma sheet. During quiet times all crossings 

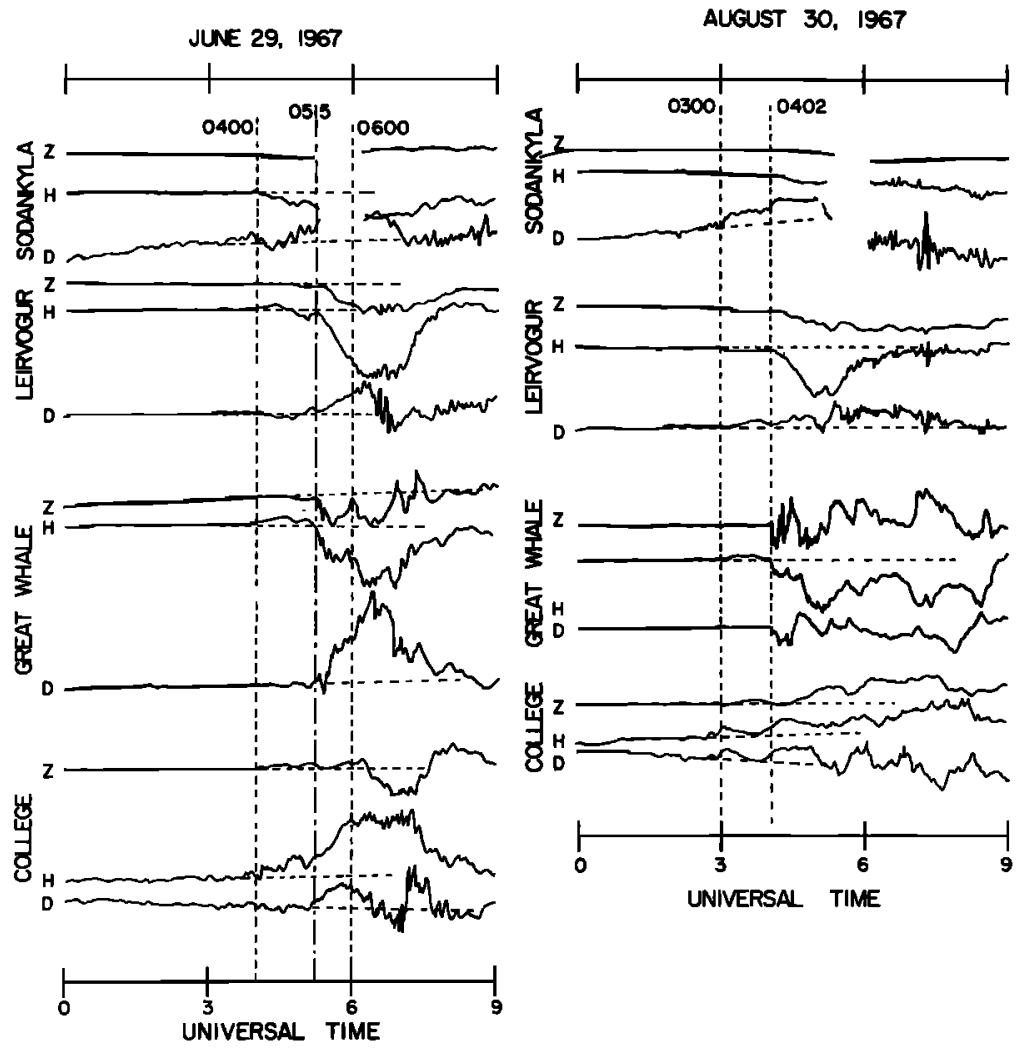

Fig. 5. Same as Figure 4 for June 29 and August 30, 1967.

of this boundary occurred at distances of approximately $11 R_{s}$. However, all crossings that occurred during bay activity were at unusually close distances, some as close as $6 R_{B}$.

Carpenter and Stone [1967] have interpreted inward drifts of whistler ducts one-half hour before the expansion phase as evidence for large-scale magnetospheric electric fields. According to D. L. Carpenter (personal communication, 1968), this motion is a characteristic of substorms when observed by whistler stations within a several-hour interval around local midnight.

Freeman and Maguire [1967] have suggested that plasma is convected inward to $6.6 R_{B}$ on the night side during substorms. With a retarding potential analyzer on ATS 1 , they found order of magnitude increases in count rates in less than 15 min during magnetic bays.

From ground measurements of VLF Morozumi and Helliwell [1966] suggested that there exists a ' $N 1$ phase' prior to the auroral breakup or expansion phase of a substorm. Their $N 1$ phase is characterized by large-amplitude unstructured hiss. The amplitude of the hiss appears impulsive on a time scale of minutes. The duration of this phase is from $10 \mathrm{~min}$ to 3 hours.

Micropulsations as well as VLF hiss have been reported as a precursor to the expansion phase of substorms. Rostoker [1968] has reported that bursts of $\mathrm{Pi} 2$ micropulsations occur before the beginning of the expansion phase as well as coincident with it. The author $[M c$ Pherron, 1968] has found that these bursts occur with increasing probability throughout the growth phase.

These bursts appear to be related to activation of auroral arcs [Victor, 1965], energetic electron precipitation [Heacock, 1967; McPherron et al., 1968], and perturbations on standard magnetograms (trigger bays) [Rostoker, 1968].

All these reports substantiate the interpreta- 
tion made in this paper, i.e., that a magnetospheric substorm has a significant growth phase. The characteristics of this phase are briefly summarized below.

Summary of growth phase characteristics. Significant deviations of the magnetic field from its quiet values occur before the expansion phase of magnetospheric substorms. These deviations are observed on the ground in the auroral zone and at a satellite in a synchronous equatorial orbit when it is located within a few hours of local midnight. The ground disturbances are positive and negative bays characteristic of the local time of the observatory, whereas the disturbance at the satellite consists of a depression in the $H$ component. A magnetospheric electric field at distances of $4 R_{E}$ on the night side appears to be associated with the growth phase near the midnight meridian. Also, injection of plasma may be occurring during this phase. It is likely that the plasma injection is related to inward motion of the low-energy electron boundary. Micropulsation noise bursts associated with activation of portions of auroral arcs, precipitation of energetic electrons, VLF hiss, and bay-like depressions of short duration are observed during the growth phase. This phase is terminated by the expansion phase, which at the synchronous orbit is characterized by sudden recovery in the $H$ component and large increases in the fluxes of energetic trapped electrons.

Interpretation of growth phase. Magnetospheric convection has been suggested as the cause of polar magnetic substorms [e.g., Axford, 1969]. In this model electric fields associated with the convection are transmitted to the auroral ionosphere via magnetic field lines where they drive ionospheric currents. Since the magnetic variations during the growth phase are similar to those in the expansion phase, it is quite likely that the current systems are the same. Thus, we suggest that the start of the substorm indicates either a commencement or a gradual enhancement of magnetospheric convection. The electric field increases caused by this change and, perhaps, also by an increase in ionospheric conductivity due to increased precipitation would cause an increase in ionospheric currents. These currents in turn would cause gradual departures of various components from quiet base lines.
The remainder of the substorm growth phase may then continue as described by Axford [1969]. Flux builds up in the tail because inward convection becomes blocked by plasma previously convected into it and by ionospheric line-tying enhanced by increased precipitation. The growth phase is terminated when, for as yet unknown reasons, magnetic field lines at the inner edge of the cusp collapse inward and dump particles into the ionosphere. A sudden increase in conductivity results, which causes a dramatic increase in the magnitude of ionospheric currents already flowing during the growth phase.

In conclusion, we would like to emphasize that it is not necessary to postulate a new current system to explain the magnetic variations observed during the growth phase. In fact, the assumption that this current system is nearly the same as that during the expansion phase is consistent with the ideas discussed by Axford and others, i.e., that a substorm expansion is preceded by enhanced inward magnetospheric convection. In this regard, we note that, although we have used only isolated substorms, it likely that substorms late in a sequence of substorms also have a growth phase, i.e., a period of enhanced inward convection following the collapse of a previous substorm. Identification of these growth phases with auroral zone magnetograms is much more subjective than the identification for the events discussed above.

Acknowledgments. I wish to thank Professor S. H. Ward for his interest and support while much of this work was conducted. Many of the ideas suggested herein have been discussed freely among my colleagues, including F. V. Coroniti, G. K. Parks, C. Liu, C. Kennel, W. D. Cummings, P. J. Coleman, Jr., and T. A. Farley.

Financial support for this work included NSF GA 1315 while at the University of California, Berkeley, and NASA NAS 5-9098 while at the University of California, Los Angeles.

The Editor wishes to thank G. Rostoker for his assistance in evaluating this paper.

\section{REFERENCES}

Akasofu, S.-I., The development of the auroral substorm, Planet. Space Sci., 12, 273-282, 1964. Akasofu, S.-I., S. Chapman, and C.-I. Meng, The polar electrojet, J. Atmos. Terr. Phys., 27, $1275-1305,1965$.

Akasofu, S.-I., C-I. Meng, and D. S. Kimball, 
Dynamics of the aurora, 4, Polar magnetic substorms and westward traveling surges, J. Atmos. Terr. Phys., 28, 489-496, 1966a.

Akasofu, S.-I., D. S. Kimball, and C.-I. Meng, Dynamics of the aurora, 5, Poleward motions, J. Atmos. Terr. Phys., 28, 497-503, 1966 .

Akasofu, S.-I., and C.-I. Meng, A study of polar magnetic substorms, J. Geophys. Res., 74(1), 293-313, 1969.

Axford, W. I., Magnetospheric convection, Rev. Geophys., 7, 421-459, 1969.

Brice, N., Morphology of elementary magnetospheric substorms, Conjugate Point Symposium, July, 1967, ESSA Tech. Mem. IERTMITSA $\gamma 2$, July 1967.

Carpenter, D. L., and K. Stone, Direct detection by a whistler method of the magnetospheric electric field associated with a polar substorm, Planet. Space Sci., 15, 395-397, 1967.

Coroniti, F. V., R. L. McPherron, and G. K. Parks, Studies of the magnetospheric substorms, 3, Concept of the magnetospheric substorm and its relation to electron precipitation and micropulsations, J. Geophys. Res., 79(5), 1715-1722, 1968.

Cummings, W. D., and P. J. Coleman, Jr., Simultaneous magnetic field variations at the earth's surface and at synchronous equatorial distance, 1, Bay-associated events, Radio Sci., $3(7)$, 758-761, 1968.

Cummings, W. D., J. N. Barfield, and P. J. Coleman, Jr., Magnetospheric substorms observed at the synchronous orbit, J. Geophys, Res., $73(21), 6687-6698,1968$.

Freeman, J. W., Jr., and J. J. Maguire, Gross local-time particle asymmetries at the synchronous orbit altitude, J. Geophys. Res., 7\%(21), 5257-5264, 1967.

Heacock, R. R., Two subtypes of type Pi micro- pulsations, J. Geophys. Res., 7Q(15), 3905-3917, 1967.

McPherron, R. L., Relation of auroral zone micropulsations to magnetospheric substorms, $\mathrm{Ph} . \mathrm{D}$. dissertation, University of California, Berkeley, September 1968.

McPherron, R. L., G. K. Parks, and F. Coroniti, Relation of correlated magnetic micropulsations and electron precipitation to the auroral substorm, Conjungate Point Symposium, July 1967, ESSA Tech. Mem. IERTM-ITSA 7Q, July 1967.

McPherron, R. L., G. K. Parks, F. V. Coroniti, and S. H. Ward, Studies of the magnetospheric substorm, 2, Correlated magnetic micropulsations and electron precipitation occurring during auroral substorms, J. Geophys. Res., $73(6)$, 1697-1713, 1968.

Meng, C.-I., and S.-I. Akasofu, Intense negative bays inside the auroral zone, 2, Indented positive bays, J. Atmos. Terr. Phys., 29, 13051310, 1967.

Morozumi, H. M., and R. A. Helliwell, A correlation study of the diurnal variation of upper atmospheric phenomena in the southern auroral zone, Sci. Rep. \&, SU-SEL-66-124, Stanford Electronics Laboratories, December 1966.

Rostoker, G., Macrostructure of geomagnetic bays, J. Geophys. Res., $\gamma S(13), 4217-4229,1968$.

Vasyliunas, V. M., A survey of low-energy electrons in the evening sector with OGO 1 and OGO 3, J. Geophys. Res., 73(9), 2839-2884, 1968.

Victor, L. J., Correlated auroral and geomagnetic micropulsations in the period range 5 to 40 seconds, J. Geophys. Res., 7O(13), 3123-3130, 1965.

(Received April 20, 1970.) 